\title{
Caracterización del desempeño en actividades de la vida diaria en adultos con lesión de miembro superior ${ }^{1}$
}

\section{Characterization of Performance in Activities of Daily Life of Adults with Injuries of Upper Limb}

\author{
Andrea Estefanía Bernal Salamanca ${ }^{2}$ \\ Diana Marcela Fuquen Fraile 3 \\ Edwin Alfonso Valenzuela Murillo ${ }^{4}$ \\ Sylvia Cristiana Duarte Torres ${ }^{5}$
}

Recibido: Septiembre 122013 • Enviado para modificación: Noviembre 92013 • Aceptado: Junio 52014

Bernal, A.; Fuquen, D.; Valenzuela, E. \& Duarte, S. (2014). Caracterización del desempeño en actividades de la vida diaria en adultos con lesión de miembro superior. Revista Ocupación Humana, 14 (1), pp. 5-16.

Resumen: La alteración de la independencia y la autonomía en actividades de la vida diaria (AVD) de adultos con alguna lesión de miembro superior de origen periférico, es un tema del cual se evidencia un abordaje mínimo de acuerdo a los antecedentes investigativos revisados. El objetivo de la investigación es caracterizar el desempeño ocupacional de usuarios con lesión de miembro superior en la ejecución de tareas específicas en las actividades de la vida diaria. Se realiza un estudio descriptivo de tipo trasversal, a través de la aplicación del Test de Actividades de la Vida Diaria -TAVD (Arango y Rodríguez, 1994) a 84 adultos, de los cuales se obtuvieron resultados para la caracterización de su desempeño de acuerdo a las variables de edad, género, tiempo de evolución y ocupación. Los resultados muestran que la actividad de vestido y las tareas de abotonarse, amarrar zapatos y vestir y desvestir un pantalón, constituyen las de mayor alteración. Así mismo, los participantes en condición de amputación y lesión tendinosa presentan mayores dificultades en estas actividades. La investigación aporta información valiosa para soportar el ejercicio del terapeuta ocupacional y guiar su intervención hacia las principales dificultades que se pueden encontrar en la población abordada.

Palabras clave: Actividades cotidianas, extremidad superior, lesiones músculo esqueléticas, adulto, Terapia Ocupacional.

Abstract: Alteration of independence and autonomy in activities of daily life (ADL) of adults
with an injury of upper limb of peripheral origin is a subject of which there is evidence of a
minimum approach according to the revised investigative background. The objective of the
research is to characterize the occupational performance of users with upper limb injury in
performing specific tasks during the daily life activities. Is a descriptive study of transverse

\footnotetext{
${ }^{1}$ Artículo basado en el trabajo de grado, realizado por los autores para optar por el título de terapeutas ocupacionales de la Universidad Nacional de Colombia bajo la dirección de la T.O. Silvia Cristina Duarte Torres.

${ }^{2}$ Estudiante de Terapia Ocupacional. Universidad Nacional de Colombia. anebernalsa@unal.edu.co

${ }^{3}$ Estudiante de Terapia Ocupacional. Universidad Nacional de Colombia.dmfuquenf@unal.edu.co

${ }^{4}$ Estudiante de Terapia Ocupacional. Universidad Nacional de Colombia. eavalezuelam@unal.edu.co

${ }^{5}$ Terapeuta ocupacional. Magister en Bioética. Docente asociada, Departamento de la Ocupación Humana. Universidad Nacional de Colombia.scduartet@unal.edu.co
} 
type, through the application of the Daily Life Activities Test - TAVD (Arango y Rodríguez, 1994) to 84 adults, which results in the characterization of their performance according to the variables of age, gender, time of evolution and occupation. The results shows that dress activity and the tasks of buttoning, tying shoes, dress and undress trousers are those of greater alteration. Likewise, participants with amputation and tendon injury presented major difficulties in these activities. The research provides information that supported the exercise of the occupational therapist and guide its intervention towards the main difficulties encountered in the population addressed.

Key words: Daily living activities, upper extremity, musculoskeletal injuries, adult, Occupational Therapy.

\section{Introducción}

Las actividades de la vida diaria (AVD), según el marco de trabajo de Terapia Ocupacional, son definidas como las "actividades orientadas al cuidado del propio cuerpo" (Rogers y Holm, 1994; citado por AOTA, 2010, p. 10). El área de autocuidado establece, en primera instancia, la forma de supervivencia del ser humano durante todas las etapas del ciclo vital, tomando un lugar predominante dentro de la Ocupación Humana, lo que permite estructurar el cimiento de la autonomía y la independencia personal. Estas actividades constituyen una de las áreas ocupacionales (autocuidado, juego y trabajo), en la cual se refleja la independencia con la que un individuo puede ejecutar tareas de manera funcional para el cuidado de sí mismo.

En este sentido, a medida que un individuo se desempeña de manera efectiva en cada una de sus áreas ocupacionales, se refleja también la funcionalidad de sus componentes del desempeño, necesarios para la ejecución de las diferentes actividades. Las lesiones músculo-esqueléticas son ejemplos claros de las alteraciones en el desempeño que a lo largo del ciclo vital, pueden presentarse y limitar principalmente el estado de independencia de la persona en AVD en el área de autocuidado.

Una lesión de miembro superior se define como una alteración funcional debida a una enfermedad o herida en las extremidades superiores. El traumatismo y la enfermedad del sistema músculo esquelético varían desde lesiones súbitas, agudas y fatales hasta cambios graduales en la función por el deterioro de las articulaciones, los nervios y el tejido conectivo. Pueden presentarse efectos secundarios como contracturas y atrofia muscular, limitaciones de la movilidad articular, deformidad en la alineación, debilidad, disfunción sensitiva y dolor crónico (Willard, Crepeau, Spackman, Cohn \& Schell, 2005).

En Colombia los desórdenes músculo esqueléticos relacionados con el trabajo han cobrado particular relevancia en la última década, teniendo en cuenta las características de la morbilidad profesional publicadas por el Ministerio de Protección Social (2008). Es así que entre los años 2001 y 2005, la primera causa de morbili- 
dad profesional fueron los desórdenes músculo esqueléticos, siendo el síndrome de túnel del carpo el de mayor prevalencia. Adicionalmente, entre los desórdenes músculo esqueléticos de origen común (no relacionadas con el trabajo), sobresale la artritis reumatoide en la población latinoamericana, con un $0.5 \%$ afectados, es decir, entre 250.000 y 450.000 personas sufren de esta enfermedad crónica (Delgado y Cols, 2006; citado por Quiceno \& Vinaccia, 2011).

Los referentes epidemiológicos que dan cuenta de la prevalencia de trastornos músculo-esqueléticos y enfermedades articulares en la población adulta colombiana, sugieren la intervención oportuna de Terapia Ocupacional con miras a mantener, compensar o restaurar las habilidades necesarias para el desempeño en las AVD. Para ello se hace imprescindible contar con instrumentos de evaluación que proporcionen una base de información sólida, veraz y específica, que permita precisar las verdaderas necesidades del individuo y elegir el mejor programa de tratamiento que procure en la persona un mejor desempeño en esta área.

El objetivo de la investigación es entonces caracterizar el desempeño ocupacional de personas con lesión de miembro superior en la ejecución de tareas específicas en las AVD.

\section{Método}

Se realizó un estudio descriptivo de tipo transversal para caracterizar el desempeño en las AVD de un grupo adultos con diferentes tipos de lesión de miembro superior según las varia- bles género, edad, tiempo de evolución de la lesión y ocupación. Este estudio se llevó a cabo en el Laboratorio de Autonomía del Departamento de la Ocupación Humana de la Universidad Nacional de Colombia- Sede Bogotá, bajo condiciones homogeneizadas en cuanto a lugar, tiempo y materiales utilizados.

Para describir el desempeño en AVD existen varios instrumentos a nivel internacional y nacional, como el Índice de Barthel (Mahoney, 1965; citado por Ruzafa \& Moreno, 1997), el Índice de Katz (Katz, Ford, Moskowitz, Jackson y Jaffe, 1963), la Medida de Independencia Funcional (Martínez, Fernandez, Frades, Rojo, Petidier, Rodríguez, Forjaz, Prieto y Cuesta, 2009), la Evaluación de Habilidades de la Vida de Kohlman (Gómez, 2001), la Escala de Actividades de la Vida Diaria de KleinBell (Klein y Bell, 1979; citado por Dahlgren, Sand, Larsson, Karlsson y Claesson, 2013), entre otros. Para este estudio se decide utilizar el Test de Actividades de la Vida Diaria (TAVD) (Arango y Rodríguez, 1994, 1995) debido a su creación y validación en el contexto colombiano, su tiempo de aplicación -30 minutos aproximadamente-, su metodología de observación directa al individuo en lugar de entrevista y lista de chequeo, la familiaridad de los objetos utilizados con la población colombiana, así como el desglosamiento de las tareas evaluadas.

El TAVD permite evaluar el desempeño funcional de la población adulta colombiana a través de 52 tareas en siete actividades básicas (Tabla 1), reconociendo el grado de independen- 
cia-dependencia en sus realizaciones cotidianas y permitiendo determinar qué tipo de actividades se alteran con mayor frecuencia, según el nivel del segmento corporal afectado, la edad, y el tipo de alteración. Su calificación se da según el nivel de desempeño de la siguiente forma: 0 , dependencia; 1 , semi-dependencia; 2 , semi- independencia; y 3 independencia.

Para asegurar la confiabilidad de los resultados, se realizó un entrenamiento previo de los investigadores con personas que presentaban diferentes diagnósticos de lesión de miembro superior, el cual tuvo una duración de un semestre.

Tabla 1. Clasificación de actividades de la vida diaria del TAVD

\begin{tabular}{l}
\multicolumn{1}{c}{$\begin{array}{c}\text { Actividad } 1 . \\
\text { Movilidad en cama }\end{array}$} \\
Acostarse y levantarse de la \\
cama \\
Acomodar almohada y cobija \\
Adoptar posición cómoda \\
Rolar \\
Moverse de cabecera al pie de \\
la cama \\
Tomar objetos de la mesa de \\
noche \\
Adoptar posición semi-acostado \\
Sentarse en la cama \\
Sentarse al borde de la cama \\
Desplazarse en posición \\
sentada
\end{tabular}

Actividad 2. Vestido

Brassier/ ropa interior / pañal

Camisa / chaqueta

Camisa/ buzo/ vestido

Pantalón / falda

Media / media pantalón

Zapato / cordón / mocasín /

bota

Botón / cremallera / velcro

Acceso: reloj/anillo/cinturón/

otro

\section{Actividad 3.}

Alimentación

Verter líquidos

Pasar solidos de un plato a otro Manipular utensilios en el área

Come: Dedos/cuchara/tenedor/ cuchillo

Bebe: Vaso/pocillo/ taza/ pitillo

\section{Actividad 4. Movilidad en cama}

Talco/desodorante/ posición Limpieza de nariz

Limpieza de uñas: manos/pies

Afeitado/cuidado de piel/

maquillaje

Peinar cabello

\section{Actividad 5. Higiene personal}

Bañar/secar manos

Bañar/secar cara

Lavar cabello

Bañar/ secar tronco y miembros superiores

Bañar/secar miembros inferiores

Colocar/retirar toalla femenina

Bañar/secar espalda

Bañar/secar pies

Limpieza de dientes/prótesis

\section{Actividad 6.}

Ir al baño

Uso: sonda/pañal/catéter/pato/ inodoro

Manejo de ropas antes, después

del acto

Adoptar la posición

Limpieza del periné

Organización del Medio: pañal/

catéter/pato/ inodoro

\section{Actividad 7. Traslados funcionales}

De la cama al piso/Silla de ruedas/camilla

Entrar a la ducha/tina

Salir del cuarto

Subir escaleras

Bajar escaleras

Subir rampas

Bajar rampas

Sentarse en una silla

Levantarse de una silla

Moverse con carga

Esquivar barreras arquitectónicas

Fuente. Adaptado por los autores de Arango y Rodríguez (1994, 1995). 
La población de interés fueron adultos con algún tipo de lesión de miembro superior de origen periférico que cumplieran con los siguientes criterios de inclusión: 1. Estar ubicado dentro de las etapas del ciclo vital conocidas como adultez temprana, intermedia o tardía. 2. Haber sido diagnosticado por médico general o especialista con algún tipo de lesión de miembro superior de origen periférico.

Los criterios de exclusión fueron: 1 . Tener compromiso neurológico que altere el tono muscular de miembros superiores. 2. Comorbilidad con enfermedades crónicas que deterioren notablemente la calidad de vida y alteren la aplicación y los resultados del test, tales como: Cáncer, SIDA, EPOC, entre otras. 3. Manifestar de forma verbal, antes o durante la aplicación del test, el deseo de no continuar dentro de la investigación, retirando el consentimiento informado.
La población se contactó a través de un centro de rehabilitación de miembro superior, una aseguradora de riesgos laborales, la Dirección de Bienestar Universitario de cada facultad de la Universidad Nacional de Colombia y finalmente, familiares y amigos que cumplieran con los criterios de inclusión y exclusión.

Se contó con 84 adultos, los cuales fueron caracterizados de acuerdo a las variables: género, edad en grupos etarios (Papalia, Olds y Feldman, 2005), tipo de lesión (de acuerdo con la Clasificación Internacional de Enfermedades de la OMS, 1992), tiempo de evolución (según la Guía de Atención Integral Basada en la Evidencia para Desórdenes Músculo esqueléticos del Ministerio de Protección Social, 2006) y ocupación (acorde con la Clasificación Internacional Uniforme de las Ocupaciones adaptada para Colombia por el DANE, 2005).

Tabla 2. Caracterización de la población estudiada

\begin{tabular}{|c|c|c|c|c|c|c|c|c|c|c|}
\hline \multicolumn{2}{|r|}{ Tipo de lesión } & 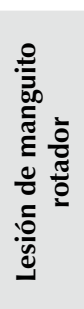 & 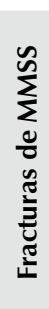 & 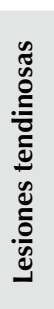 & 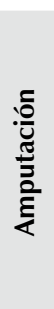 & 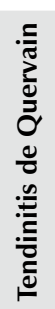 & 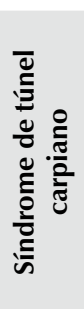 & 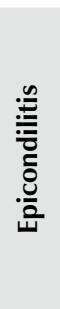 & 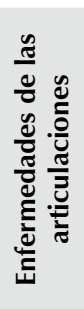 & $\frac{1}{\sigma}$ \\
\hline \multicolumn{2}{|c|}{$\begin{array}{l}\text { Personas por tipo de } \\
\text { lesión }\end{array}$} & 17 & 17 & 9 & 7 & 6 & 10 & 10 & 8 & 84 \\
\hline \multicolumn{11}{|c|}{ Variables } \\
\hline \multirow{2}{*}{ 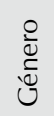 } & Mujeres & 10 & 3 & 3 & 0 & 6 & 7 & 7 & 8 & 44 \\
\hline & Hombres & 7 & 14 & 6 & 7 & 0 & 3 & 3 & 0 & 40 \\
\hline
\end{tabular}


(Continuación Tabla 2)

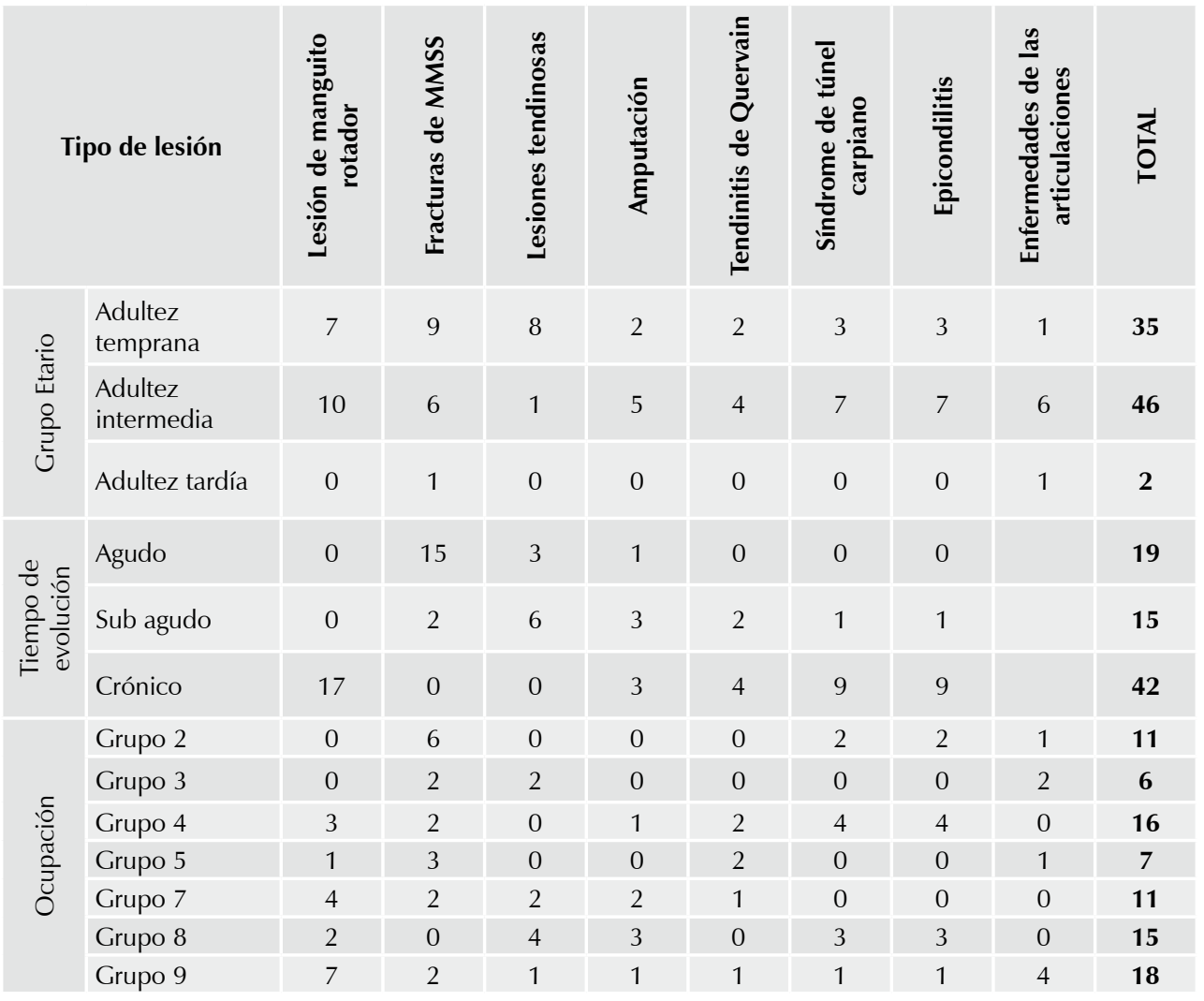

Fuente. Elaboración propia.

La tabla 2 muestra la caracterización del grupo; en la Figura 1, se presenta la distribución porcentual de los participantes de acuerdo al tipo de lesión.

Figura 1. Distribución de los participantes según tipo de lesión - diagnósticos

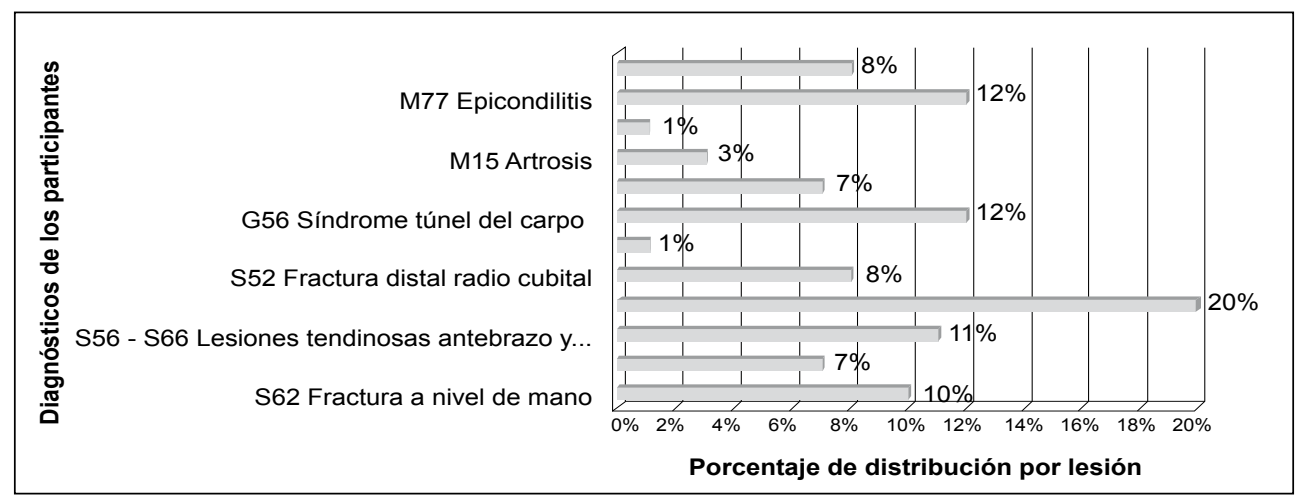

Fuente. Elaboración propia. 


\section{Consideraciones éticas}

La presente investigación tuvo en cuenta las disposiciones generadas por los estamentos legales y científicos a nivel nacional e internacional, para la protección de los derechos de las personas involucradas en el proceso investigativo. En este sentido, previo al inicio de la recolección de los datos, se informó a cada participante los alcances del estudio, la metodología de la evaluación, el propósito del mismo y el manejo de los datos generados en la evaluación. Así mismo, se explicó que la participación era voluntaria, sin ningún tipo de remuneración económica. Posterior a esto, cada participante manifestó de forma escrita su decisión voluntaria y libre de participar en la investigación, firmando el consentimiento diseñado por los autores.

\section{Resultados}

Este apartado describe los resultados de la aplicación del TAVD (Arango y Rodríguez, 1994) para cada grupo de adultos, de acuerdo al tipo de lesión y teniendo en cuenta las variables antes mencionadas, teniendo como referencia que el instrumento de evaluación utilizado consta de una escala de puntuación que oscila entre cero (porcentaje mínimo de desempeño) y 100 (porcentaje máximo de desempeño).

Grupo de personas con lesiones de manguito rotador. La actividad en la que se presentan mayores dificultades en la ejecución para este grupo es la movilidad en cama, con un $72,9 \%$ de promedio de ejecución entre las personas evaluadas, seguido de la actividad de acicalado, con un $84,7 \%$ y la actividad de vestido con un $85,7 \%$.

Las tareas en la actividad de movilidad en cama, en las cuales se presenta mayor dificultad, son aquellas relacionadas con adoptar la posición semi-acostado, seguida de la tarea de rolar y moverse de la cabecera al pie de la cama, las cuales se ven representadas por presentar mayor calificación cero (0) dentro del grupo evaluado. (Figura 2).

Figura 2. Distribución porcentual según tarea con menor desempeño en la actividad movilidad en cama. Grupo de adultos con lesiones de manguito rotador

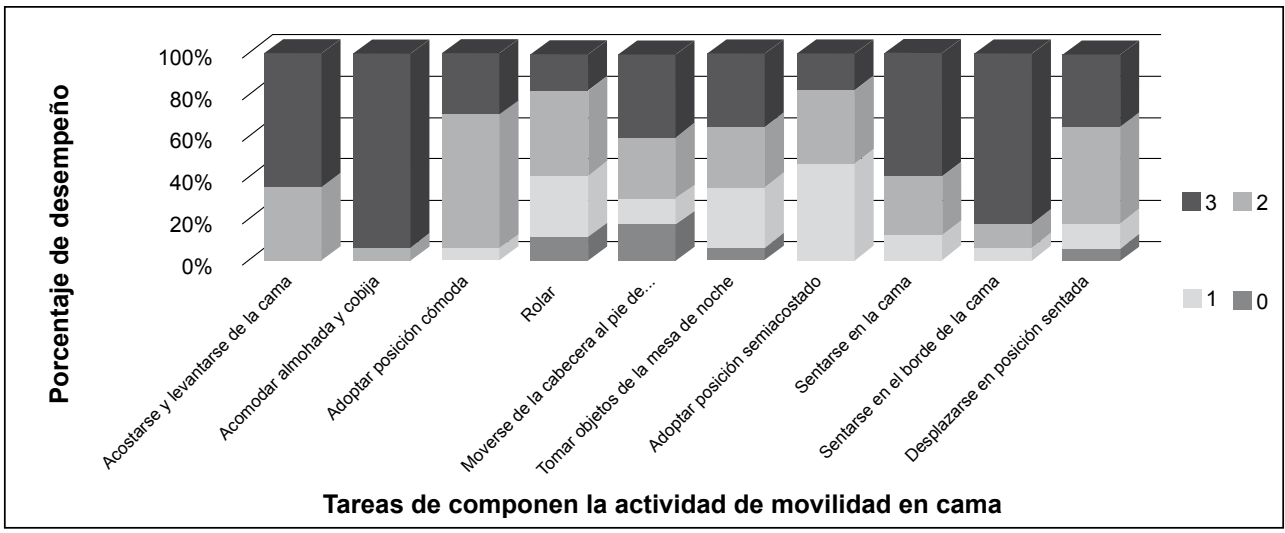

Fuente. Elaboración propia. 
Grupo de personas con lesiones tendinosas. Para las personas con lesión tendinosa de antebrazo y mano, la actividad en la que se presentan mayores dificultades es la de vestido, con un $72,2 \%$ de promedio de ejecución entre las personas evaluadas.

Las tareas en la actividad de vestido en las cuales se presenta mayor dificultad, son aquellas relacionadas con vestir y desvestir pantalones y faldas, seguida de abotonar, subir cremalleras y despegar velcros; estas presentan con mayor frecuencia la calificación cero $(0)$ en el caso de la primera tarea y calificaciones menores a tres en la segunda.

Grupo de personas con fracturas de miembro superior. La actividad en que se presentan mayores dificultades para este grupo corresponde a la alimentación, con un $76,87 \%$ de promedio de ejecución entre las personas evaluadas, seguido de la actividad de acicalado, con un $77,65 \%$, y la actividad de vestido con un $78,93 \%$.

La tarea en la cual se presenta mayor dificultad para ser ejecutada tras una fractura de miembro superior es manipular utensilios en el área, seguida de la tarea de comer con cubiertos.

\section{Grupo de personas con amputación} de miembro superior. Para este grupo la actividad en que se presentan mayores dificultades es la de vestido, con un $60,71 \%$ de promedio de ejecución.

La tarea en la cual se presenta mayor dificultad tras una amputación de miembro superior es la tarea de abotonar, subir y bajar cremalleras y cerrar velcros.

Grupo de personas con artritis. La actividad en que se presentan mayores dificultades para la población con artritis y artrosis corresponde a la actividad de vestido, con un $80,76 \%$ de promedio de ejecución entre las personas evaluadas, seguido de la actividad de acicalado con un $84,17 \%$ y la actividad de alimentación con un $85,84 \%$.

La tarea de mayor dificultad fue colocar camiseta, buzo o vestido, seguida de colocar zapatos y amarrar cordones.

Grupo de personas con tendinitis de Quervain. Se presenta mayor dificultad en las actividades de alimentación y acicalado, con un promedio de $83 \%$ de ejecución entre las personas evaluadas. La mayor dificultad se da en las tareas de peinar cabello y limpieza de uñas.

Grupo de personas con síndrome de túnel del carpo. De acuerdo con los resultados del test, se presenta mayor dificultad en el área de vestido, con un promedio de $75 \%$ de ejecución entre las personas evaluadas, seguido de la actividad de acicalado con un $77 \%$ y las actividades de movilidad en cama y alimentación con un $84 \%$.

Las tareas de mayor dificultad son apuntar botón, subir cremallera y ponerse pantalón o falda.

Grupo de personas con síndrome de epicondilítis. En este grupo se presenta mayor dificultad en la actividad de acicalado, con un promedio de $70 \%$ de ejecución entre las personas evaluadas. Se presenta mayor dificultad en las tareas de peinar cabello y aplicar talco o desodorante.

Teniendo en cuenta los anteriores resultados, la Tabla 3 resume las actividades y tareas más afectadas según el tipo de lesión. 
Tabla 3. Actividades y tareas de la vida diaria con menor desempeño según tipo de lesión

\begin{tabular}{|c|c|c|c|}
\hline Tipo de lesión & $\begin{array}{c}\text { Porcentaje } \\
\text { de desempeño }\end{array}$ & $\begin{array}{l}\text { Actividad } \\
\text { con menor } \\
\text { desempeño }\end{array}$ & $\begin{array}{l}\text { Tareas con mayor dificultad } \\
\text { de ejecución }\end{array}$ \\
\hline $\begin{array}{l}\text { Lesiones de } \\
\text { manguito rotador }\end{array}$ & $72,9 \%$ & Movilidad en cama & $\begin{array}{l}\text { Adoptar posición semi-acostado. } \\
\text { Moverse de la cabecera al pie de la } \\
\text { cama. }\end{array}$ \\
\hline Lesiones de tendones & $72,2 \%$ & Vestido & $\begin{array}{l}\text { Vestir y desvestir pantalón o falda. } \\
\text { Abotonar, subir cremallera, } \\
\text { desprender velcros. }\end{array}$ \\
\hline $\begin{array}{l}\text { Fracturas en } \\
\text { miembro superior }\end{array}$ & $76,8 \%$ & Alimentación & $\begin{array}{l}\text { Manipular utensilios en el área } \\
\text { Pasar sólidos de un plato a otro. }\end{array}$ \\
\hline $\begin{array}{l}\text { Amputaciones en } \\
\text { miembro superior }\end{array}$ & $60,7 \%$ & Vestido & $\begin{array}{l}\text { Abotonar, subir cremallera, } \\
\text { desprender velcros. } \\
\text { Poner zapatos, amarrar cordones, } \\
\text { poner botas. }\end{array}$ \\
\hline $\begin{array}{l}\text { Tendinitis de } \\
\text { Quervain }\end{array}$ & $83 \%$ & Acicalado & $\begin{array}{l}\text { Peinar cabello. } \\
\text { Limpieza de uñas de manos y de } \\
\text { pies. }\end{array}$ \\
\hline $\begin{array}{l}\text { Síndrome de túnel } \\
\text { del carpo }\end{array}$ & $75 \%$ & Vestido & $\begin{array}{l}\text { Abotonar, subir cremallera, } \\
\text { desprender velcros. } \\
\text { Vestir y desvestir pantalón o falda. }\end{array}$ \\
\hline Epicondilitis & $70 \%$ & Acicalado & $\begin{array}{l}\text { Peinar el cabello. } \\
\text { Aplicar talco y desodorante. }\end{array}$ \\
\hline $\begin{array}{l}\text { Enfermedades de las } \\
\text { articulaciones }\end{array}$ & $81 \%$ & Vestido & $\begin{array}{l}\text { Colocar camiseta, buzo y vestido. } \\
\text { Amarrar zapatos. }\end{array}$ \\
\hline
\end{tabular}

Fuente. Elaboración propia.

\section{Discusión}

La presente investigación provee indicios importantes respecto a las alteraciones en las AVD en adultos con diferentes tipos de lesión de miembro superior.

La información generada se constituye en una aproximación para futuros estudios, que abarcando muestras representativas de la población por tipo de lesión, puedan generar resultados generalizables, proporcionando así una evidencia aplicable a los programas de prevención y rehabilitación de Terapia Ocupacional. Así por ejemplo, en la
Guía Técnica del Sistema de Vigilancia Epidemiológica en Prevención de Desórdenes Músculo-esqueléticos en Trabajadores en Colombia (Ministerio de Protección Social, 2008), las AVD, como componente esencial del ámbito extralaboral, no aparecen contempladas dentro de los programas básicos de seguridad y salud en el trabajo, aun cuando este tipo de desórdenes, como se aprecia en los resultados del estudio, tienen una influencia importante en la posibilidad de las personas de realizar sus actividades cotidianas con el mayor grado de bienestar físico y mental. 
En esta misma línea, los resultados por nivel de lesión, tipo de lesión y las variables edad, género, ocupación y tiempo de evolución aquí contempladas, apoyan los resultados de los estudios de Yeung (2009) y Ortega (2003), en los cuales se reporta una correlación entre el desempeño en AVD y el síndrome del túnel del carpo y la epicondilitis, lo cual sugiere que a pesar de la presencia de deterioro fisiológico leve, moderado o grave en las diferentes regiones del miembro superior aquí evaluadas, las limitaciones en el desempeño ocupacional están presentes y pueden ser un indicador temprano de la patología.

En este sentido, la presente investigación se constituye en una aproximación que soporta el ejercicio profesional del terapeuta ocupacional y guía su intervención hacia las principales dificultades que se pueden encontrar en personas con lesiones de miembros superiores de origen periférico. Se extiende de esta manera la invitación a contemplar la repercusión de este tipo de actividades dentro de diferentes escenarios ocupacionales donde se desempeña el ser humano.

Sin embargo, se debe tener en cuenta que el TAVD (Arango y Rodríguez, 1994), no considera para su valoración diferencias culturales de la población colombiana, seccionando la población de estudio a participantes que se desenvuelven o tienen conocimiento de contextos urbanos, dejando de lado diferentes características y adaptaciones de la tareas, locaciones y hábitos de zonas rurales.
Las limitaciones de la investigación incluyen: un grupo de estudio con pocos representantes de las diferentes patologías abordadas, $(6<\mathrm{n}<$ 17) y poca variedad en los tiempos de evolución de los diagnósticos contemplados. Lo anterior se deriva del tiempo dispuesto para la convocatoria de los participantes, condicionada a los tiempos exigidos en una investigación de pregrado; aun así, el estudio tiene gran relevancia, al ser un punto de partida para la creación de conocimiento a través estudios posteriores.

\section{Conclusiones}

Este estudio aporta información para la prevención de alteraciones del desempeño y la intervención que el terapeuta ocupacional puede llevar a cabo con esta población, a partir de los resultados obtenidos en torno a las características de la población, el nivel y tipo de lesión y si esta afecta o no la dominancia manual de la persona.

Los resultados muestran que la actividad de vestido, específicamente las acciones de abotonado, amarrar zapatos y vestir y desvestir un pantalón, constituyen las tareas más alteradas. Los participantes en condición de amputación y lesiones tendinosas presentan mayores dificultades en estas actividades.

Los resultados revelan una alteración significativa en actividades específicas; por ejemplo, la dificultades que presentan hombres y mujeres con lesión en el manguito rotador para llevar a cabo la movilidad en cama; la dificultad en acicalado en el grupo de 
personas con tendinitis de Quervain y epicondilitis; abotonarse para el grupo de personas con Lesiones tendinosas, enfermedades de las articulaciones, síndrome de túnel del Carpo y amputaciones; y las dificultades en alimentación en el grupo de personas con fracturas. Además, se resalta como hallazgo central que en personas con lesiones de miembro superior, la actividad de vestido es la que presenta mayor compromiso a nivel ocupacional, al ser una actividad de tipo bimanual, que requiere de tareas de manipulación sostenida de objetos y accesorios a través de patrones de agarres funcionales e integrales como lo son las pinzas.

Adicionalmente, se observó que en la población masculina es más frecuente la lesión de manguito rotador bilateral, lo cual podría relacionarse con el tipo de ocupaciones desempeñadas por los hombres, que para el grupo estudiado, se caracteriza por la manipulación de maquinaria, carga y descarga de elementos, operadores de instalaciones, manejo de herramientas, entre otros. Entre tanto en la población femenina fueron más frecuentes las lesiones unilaterales, relacionadas con trabajos en servicios generales que incluyen actividades de limpieza, que en su mayoría, se ejecutan repetitivamente y con el accionar del miembro dominante (unimanual). También, resultó evidente que la actividad de acicalado suele afectar más a la población femenina, debido a la mayor utilización de elementos durante su arreglo personal, como productos de belleza, lo cual representa una diferencia importante con relación al género masculino.
De otra parte, los rangos de edades más frecuentes, adultez intermedia y adultez temprana, podrían relacionarse con la etapa productiva de la población colombiana y los rangos de edades que suelen contratar las empresas. Así mismo, podría pensarse que en el rango de edad entre 45 y 64 años (adultez intermedia) las personas, por la cantidad de tiempo trabajado, son más vulnerables a desarrollar enfermedades profesionales y de origen común, sería conveniente revisar estudios a este respecto.

Conocer el tipo de alteración en las AVD que se puede presentar según la clase de lesión de miembro superior, permite a los profesionales de la rehabilitación, en especial los terapeutas ocupacionales, enfocar sus recomendaciones y objetivos de intervención a aquellas tareas de mayor dificultad que, por las condiciones actuales de prestación de los servicios en las IPS, no pueden ser evaluadas objetivamente por observación y bajo un test estandarizado.

Finalmente, el TAVD de Arango y Rodríguez (1994) refleja ser un instrumento de evaluación adecuado, pues contempla las tareas básicas a evaluar en un compendio corto y su tiempo de administración es mínimo, comparado con otros instrumentos valorativos de esta área como la escala de AVD de Klein Bell o el test de AVD de Linda Kollman, pero mayor a listas de chequeo como el Índice de Barthel. En este mismo sentido, el test utilizado para la presente investigación, es desarrollado bajo las características socio demográficas y culturales de la población bogotana y sus tareas poseen ín- 
tima relación con acciones efectuadas por el miembro superior, aunque no se tienen en cuenta características y adaptaciones locativas de las tareas y hábitos de origen rural.

\section{Referencias}

Arango, M. y Rodríguez, E. (1994). Diseño confiabilidad y validez del test de actividades de la vida diaria (TAVD): Arango y Rodríguez. Tesis de pregrado. Universidad Nacional de Colombia.

Arango, M., Rodríguez, E., Trujillo, A. (1995). Diseño, confiabilidad y validez del Test de Actividades de la Vida Diaria (TAVD): Arango y Rodríguez. Ocupación Humana. 6 (1): 13-63.

AOTA (2010). Marco de trabajo para la práctica de Terapia Ocupacional: Dominio y proceso. Segunda edición. American Journal Occupational Therapy. 62: 625-683).

Dahlgren, A., Sand, A., Larsson, A., Karlsson, A. y Claesson, L. (2013). Linking the KleinBell activities of daily living scale to the International Classification of Functioning, disability and health. Journal Rehabilitation Medical. 45: 351-357.

DANE. (2005). Clasificación Internacional Uniforme de Ocupaciones Adaptada para Colombia. [Consultado el 21 de noviembre del 2012]. Disponible en: http://www. dane.gov.co/index.php?option $=\mathrm{com}$ wrapper\&view $=$ wrapper\&ltemid $=144$

Gómez, A. (2001). Validación y estandarización de la evaluación de actividades de la vida diaria (AVD.) propuesta por Linda Kohlman, aplicada a personas mayores entre 60 y 80 años no institucionalizadas, en la ciudad de Bogotá. Tesis de pregrado. Universidad Nacional de Colombia.

Katz, S., Ford, A., Moskowitz, R., Jackson, B. y Jaffe, M. (1963). Studies of illness in the aged. The Index of ADL: A standardized measure of biological and psychosocial function. JAMA. 185: 914-919.
Martínez, P., Fernández, G., Frades, B., Rojo, F., Petidier, R., Rodríguez, V., Forjaz, M., Prieto, M. y Cuesta, J. (2009). Validación de la escala de independencia funcional. Gac Sanit. 23 (1): 49-54.

Ministerio de Protección Social (2006). Guía de Atención Integral Basada en la Evidencia para Desórdenes Musculo Esqueléticos (DME) relacionados con Movimientos Repetitivos de Miembros Superiores (Síndrome de Túnel Carpiano, Epicondilitis y Enfermedad de de Quervain (GATI- DME).

Ministerio de Protección Social (2008). Guía Técnica de Sistema de Vigilancia Epidemiológica en Prevención de Desórdenes Músculo Esquelético en Trabajadores en Colombia. (GT SIVEDME).

Organización Mundial de la Salud- OMS (1992). Clasificación Internacional de Enfermedades. Decima revisión.

Ortega, J. (2003). Detección de traumas acumulativos de la mano y codo en pianistas con diferente grado de experticia y su incidencia en actividades de la vida diaria. Tesis de pregrado. Escuela Colombiana de Rehabilitación.

Papalia, D., Olds, S. y Feldman, R. (2005). Desarrollo Humano. Novena Edición. México: Mc Graw-Hill.

Quiceno, J. y Vinaccia, S. (2011). Artritis reumatoide: consideraciones psicobiológicas. Revista diversitas - Perspectivas en psicología. 7 (1): 27-41.

Ruzafa, J. y Moreno, J. (1997). Valoración de la discapacidad física: el Índice de Barthel. Revista española de salud pública. 71:127-137.

Willard, H., Crepeau E., Spackman, C., Cohn, E. \& Schell, B. (2005). Terapia ocupacional.10a edición. Buenos Aires: Médica Panamericana.

Yeung, K. (2009). Hand Functioning and ADL Performance of Patients with Carpal Tunnel Syndrome (CTS) After Camitz Transfer. Hong Kong Journal of Occupational Therapy. 19(2): A7. 